\title{
Pneumothorax in HIV-infected patients: role of Pneumocystis carinii pneumonia and pulmonary tuberculosis
}

\author{
M. Tumbarello*, E. Tacconelli*, T. Pirronti**, R. Cauda*, L. Ortona*
}

\begin{abstract}
Pneumothorax in HIV-infected patients: role of Pneumocystis carinii pneumonia and pulmonary tuberculosis. M. Tumbarello, E. Tacconelli, T. Pirronti, R. Cauda, L. Ortona. (CERS Journals Ltd 1997.

ABSTRACT: Patients with acquired immune deficiency syndrome (AIDS) are at increased risk for pneumothorax, which usually occurs in the setting of Pneumocystis carinii pneumonia. The rationale of the present study was based on the hypothesis that the increased incidence of pulmonary tuberculosis in human immunodeficiency virus (HIV)-infected patients could favour the development of pneumothorax in such patients. A case-control study was performed comprising $140 \mathrm{HIV}$-infected patients grouped as follows: 35 patients with pneumothorax and 105 matched controls without pneumothorax.

Univariate analysis identified four risk factors for pneumothorax: 1) previous $P$. carinii pneumonia $(\mathrm{p}=0.01) ; 2)$ current $P$. carinii pneumonia $(\mathrm{p}=0.02)$; 3 ) pulmonary tuberculosis $(\mathrm{p}=\mathbf{0 . 0 1})$; and 4$)$ cysts, pneumatoceles or bullae on chest radiographs $(\mathrm{p}<0.001)$. Multivariate analysis indicated that current $P$. carinii pneumonia $(p=0.01)$ and pulmonary tuberculosis $(p=0.04)$ were both independent risk factors for pneumothorax.

In conclusion, our findings demonstrate that, in addition to Pneumocystis carinii pneumonia, pulmonary tuberculosis enhances the risk of pneumothorax in patients with acquired immune deficiency syndrome.

Eur Respir J 1997; 10: 1332-1335.
\end{abstract}

Depts of *Infectious Diseases and **Radiology, Catholic University, Rome, Italy.

Correspondence: M. Tumbarello Istituto Clinica Malattie Infettive Università Cattolica Sacro Cuore Largo Gemelli 8

00168 Roma

Italy

Keywords: Acquired immune deficiency syndrome

Pneumocystis carinii pneumonia

pneumothorax

tuberculosis

Received : December 101996

Accepted after revision February 241997

This work has been partially supported by the first Project on Tuberculosis (Istituto Superiore di Sanità/Ministero della Sanità, Roma).
The reported rate of spontaneous pneumothorax (PTX) in patients with acquired immune deficiency syndrome (AIDS) is $1-2 \%$, and this percentage increases to $5-10 \%$ in those patients with current or previous Pneumocystis carinii pneumonia (PCP) [1-5]. It has been hypothesized that in patients with acute PCP, the lung may rupture as a consequence of parenchymal necrosis, or formation of pneumatoceles, presumably caused by a checkvalve effect in a peripheral airway. The cysts may also be the consequence of healing and fibrosis, and, if they are overdistended, may rupture with possible PTX $[6,7]$. In addition, these pulmonary lesions may also persist after recovery from PCP, and cause PTX in some patients $[3,7]$. Cysts or bullous lung diseases have also been observed in human immunodeficiency virus (HIV)-infected patients, who have previously received aerosolized pentamidine for PCP prophylaxis and who have later developed PTX [8]. However, the occurrence of PTX in patients with PCP previously under pentamidine prophylaxis may simply reflect a complication of PCP secondary to failure of prophylaxis [9]. Rarely, PTX has also been reported in HIV-infected patients as a consequence of respiratory infections caused by agents other than P. carinii, e.g. Mycobacterium tuberculosis or Mycobacterium avium complex, Staphylococcus aureus, Streptococcus pneumoniae, Pseudomonas aeruginosa and Cytomegalovirus, or it has been associated with pulmonary Kaposi's sarcoma [2, 5].
Although the incidence of pulmonary tuberculosis, a well-recognized primary cause of spontaneous PTX in immunocompetent patients [10], has progressively increased worldwide in patients with HIV infection during the last decade [11], PTX has been surprisingly infrequently reported in these patients $[2,5]$. To further investigate the role of tuberculosis in PTX development in HIV-infected patients, we analysed the risk factors, the clinical and radiological findings and the outcome of PTX in 35 patients with AIDS, using a univariate and multivariate statistical approach.

\section{Materials and methods}

\section{Patients}

A retrospective review of the medical and radiological charts of all hospital admissions of HIV-infected patients to our ward was completed from January 1987 to December 1995. For the purpose of this study, PTX was defined as the radiographic evidence of a lung collapse. Any individual HIV-infected patient who had PTX was considered as a case. In total, 3,522 medical records of 2,691 HIV-infected patients were reviewed, and 42 episodes of PTX were identified in 38 patients. None of the patients had a history or physical evidence of 
chest trauma. One patient developed PTX after insertion of a central venous catheter and two patients (with severe PCP) were receiving continuous positive airway pressure when PTX developed. These three cases were not included in the analysis, and a final number of 39 episodes observed in 35 patients was evaluated in the study (Group A).

Each Group A patient was matched with three control HIV-infected patients ( $\mathrm{n}=105$; Group B), who had the following characteristics: 1) no evidence of PTX; 2) same sex and age (within 3 yrs); 3) hospitalization in the same ward on the month of the diagnosis of the matched case; and 4) same stage and category of HIV infection [12]. Medical records of the study patients were reviewed for the following data: HIV risk behaviour; history of opportunistic infections; time from AIDS diagnosis; antiretroviral therapy; prophylaxis for PCP; and risk factors for PTX. These latter included: intravenous drug addiction; presence of coexisting pulmonary diseases; history of aerosol pentamidine use; number of previous episodes of PCP; smoking habits (a patient was considered as a smoker if he/she had smoked more than 10 packs $\cdot$ year $^{-1}$ ); chronic obstructive pulmonary disease (COPD); and asthma.

The majority of the patients (126 out of $140 ; 90 \%$ ) were under primary or secondary PCP prophylaxis with co-trimoxazole or dapsone, or pentamidine aerosol. A patient was considered to be receiving aerosolized pentamidine if he/she had received at least three doses of the drug. In patients with current respiratory failure, the arterial oxygen tension and the fraction of inspired oxygen at the time of gas sampling were obtained. In all patients with PTX, the clinical and radiographic findings, treatment and outcome of PTX were also analysed. A recurrence was defined as the development of a new PTX more than 5 days after the recovery from the first one.

Diagnosis of PCP was made by identification of $P$. carinii cysts using an immunofluorescence assay in specimens obtained from induced sputum or bronchoalveolar lavage (BAL). Diagnosis of pulmonary tuberculosis was achieved by the isolation of $M$. tuberculosis from induced sputum or BAL. Diagnosis of bacterial pneumonia was considered if bacteria (other than Mycobacteria) were isolated from induced sputum or BAL, or from blood, in patients with respiratory symptoms and parenchymal infiltrates on chest radiographs.

On chest radiographs, PTX was defined according to the size of the collapsed lung, as: small $(<15 \%)$; moderate $(15-50 \%)$; and large ( $>50 \%$ of the collapsed lung). Air-containing pulmonary lesions with very thin walls $(<$ $2 \mathrm{~mm}$ ) were referred to as "pneumatoceles". Progression of the cysts or pneumatoceles was documented by comparison with previous chest radiographs and/or computed tomography (CT) scan.

\section{Statistical analysis}

Differences in means were tested for normal distribution and compared using Student's two-tailed t-test. Differences in group proportions were assessed using Chi-squared test or, for small numbers, using Fisher's exact test. Potential risk factors for PTX were analysed by univariate methods to screen for possible inclusion into multivariate models. Multivariate analysis was per- formed with logistic regression models, and 95\% confidence intervals (95\% CIs) were used to determinate the statistical significance of the odds ratio (OR). Multivariate analysis was performed to account for colinearity of variables, adjust for simultaneous exposures, reveal any interactions between these exposures, and determine a multivariate OR for each factor. Age and sex were not found to be confounders. The population attributable risk (PAR), a crude estimate of all PTX ascribed to pulmonary tuberculosis, was calculated as the percentage of the population risk which is attributable to the exposure. Two-tailed tests of significance at the $p<0.05$ level were used to determine statistical significance.

\section{Results}

The rate of PTX was 11.2 per 1,000 hospital admissions for HIV infection. In particular, the episode prevalence of PTX in HIV-infected patients with PCP was $9.5 \%$, and $6.8 \%$ in those with pulmonary tuberculosis. The demographic data of cases and controls are summarized in table 1. PTX occurred in 23 patients $(66 \%)$ with PCP, which was current in nine of the 23 patients $(39 \%)$ and previous in 14 out of $23(61 \%)$. Thirteen patients $(37 \%)$ suffered from pulmonary tuberculosis; 11 of the 13 patients $(85 \%)$ had tuberculosis diagnosed prior to development of PTX (median 3 months), and two patients $(15 \%)$ at the same time as PTX. One of the patients with pulmonary tuberculosis had suffered from a previous episode of PCP.

As regards the clinical findings of PTX, three patients were asymptomatic (9\%), 24 complained of chest pain (69\%), 17 dyspnoea (49\%), 14 cough (40\%), and 10 had fever $\left(>38^{\circ} \mathrm{C}\right)(29 \%)$.

\section{Statistical analysis of risk factors}

Univariate analysis identified four factors significantly associated with PTX: 1) previous PCP $(\mathrm{p}=0.01)$; 2$)$ current $\mathrm{PCP}(\mathrm{p}=0.02) ; 3)$ pulmonary tuberculosis $(\mathrm{p}=0.01)$; and 4) presence of radiological alterations of the lung, i.e. cysts, pneumatoceles or bullae on chest radiographs $(\mathrm{p}<0.001)($ table 2).

Development of PTX did not correlate with the severity of the concomitant episode of PCP, corticosteroid

Table 1. - Demographic data of 35 HIV-infected patients with PTX (cases, i.e. Group A) and 105 matched HIVinfected patients (controls, i.e. Group B)

\begin{tabular}{llc}
\hline & Group A & Group B \\
\hline Sex males/females & $32 / 3(91 / 9)$ & $96 / 9(91 / 9)$ \\
Age yrs & $33 \pm 4$ & $33 \pm 6$ \\
HIV risk factor & $25(71)$ & $79(75)$ \\
Intravenous drug abuse & $5(14)$ & $15(14)$ \\
Homosexual contacts & $4(11)$ & $10(10)$ \\
Heterosexual contacts & $1(3)$ & $1(1)$ \\
Haemophilia & $2(6)$ & $6(6)$ \\
Stage of HIV infection* & $33(94)$ & $99(94)$ \\
C2 & $58.3 \pm 63.7$ & $62.5 \pm 59.9$ \\
C3 & &
\end{tabular}

Data are presented as number of patients and percentage in parenthesis, or as mean \pm SD. *: according to the CDC classification of HIV infection [12]. HIV: human immunodeficiency virus; PTX: pneumothorax; CDC: Centers for Disease Control. 
Table 2. - Univariate analysis of risk factors for PTX: HIV-infected patients with PTX (Group A) compared to HIV-infected patients without PTX (Group B)

\begin{tabular}{|c|c|c|c|c|}
\hline $\begin{array}{l}\text { Risk factors } \\
\text { for PTX }\end{array}$ & $\begin{array}{l}\text { Group } A \\
\mathrm{n}(\%)\end{array}$ & $\begin{array}{l}\text { Group B } \\
\text { n }(\%)\end{array}$ & $\mathrm{p}$-value & $\begin{array}{c}\mathrm{OR} \\
(95 \% \mathrm{CI})\end{array}$ \\
\hline Previous PCP & $14(40)$ & $18(17)$ & 0.01 & $\begin{array}{c}3.22 \\
(1.27-8.17)\end{array}$ \\
\hline Current PCP & $9(26)$ & $10(9)$ & 0.02 & $\begin{array}{c}3.28 \\
(1.08-9.96)\end{array}$ \\
\hline $\begin{array}{l}\text { Pentamidine } \\
\text { prophylaxis }\end{array}$ & $4(11)$ & $18(17)$ & NS & - \\
\hline $\begin{array}{l}\text { Pulmonary } \\
\text { tuberculosis }\end{array}$ & 13 (37) & 17 (16) & 0.01 & $\begin{array}{c}3.05 \\
(1.18-7.88)\end{array}$ \\
\hline Bacterial pneumonia & $3(9)$ & $13(12)$ & NS & - \\
\hline $\begin{array}{l}\text { Cysts/bullae/ } \\
\text { pneumatoceles }\end{array}$ & $27(77)$ & $34(32)$ & $<0.001$ & $\begin{array}{c}7.04 \\
(2.69-18.98)\end{array}$ \\
\hline $\begin{array}{l}\text { Intravenous drug } \\
\text { addiction }\end{array}$ & $25(71)$ & $79(75)$ & NS & - \\
\hline $\begin{array}{l}\text { Pulmonary Kaposi's } \\
\text { sarcoma }\end{array}$ & $1(3)$ & $4(4)$ & NS & - \\
\hline NTM infections & $2(6)$ & $6(6)$ & NS & - \\
\hline $\begin{array}{l}\text { Smoking } \\
\quad\left(>10 \text { packs } \cdot \mathrm{yr}^{-1}\right)\end{array}$ & $18(51)$ & $59(56)$ & NS & - \\
\hline Asthma & $1(3)$ & $1(1)$ & NS & - \\
\hline COPD & $1(3)$ & $1(1)$ & NS & - \\
\hline
\end{tabular}

PTX: pneumothorax; n: number of patients; PCP: Pneumocystis carinii pneumonia; NTM: nontuberculous mycobacteria; COPD: chronic obstructive pulmonary disease; NS: nonsignificant; OR: odds ratio; $95 \%$ CI: $95 \%$ confidence interval.

treatment, number of episodes of previous PCP (one in 12 patients and two in two patients), and the time from the previous episode of PCP (mean 6.4 months, range 2-23 months). The onset of PTX was also unrelated to PCP prophylaxis with aerosolized pentamidine, HIV risk behaviour (including intravenous drug abuse), smoking habits, asthma, COPD, bacterial pneumonia, AIDSrelated diseases (such as pulmonary Kaposi's sarcoma and nontuberculous mycobacterial (NTM) infections), time from AIDS diagnosis, and antiretroviral therapy.

The logistic regression analysis indicated that, among the conditions selected by univariate analysis, only current PCP $(p=0.01 ; O R=48.42 ; 95 \% \quad C I=2.31-1015.0)$ and pulmonary tuberculosis $(\mathrm{p}=0.04 ; \mathrm{OR}=9.73 ; 95 \% \mathrm{CI}=$ 2.74-126.7) were independent risk factors for the development of PTX. The PAR for pulmonary tuberculosis to the AIDS population was $24 \%$.

\section{Radiology}

On chest radiographs, five episodes were characterized by small (14\%), 13 by moderate $(37 \%)$ and 13 by large $(37 \%)$ PTX. In the remaining four episodes (11\%), tension PTX occurred, determining a shift of the heart and mediastinum to the contralateral side, with a flattening of the cardiomediastinal contour and a depression of the ipsilateral hemidiaphragm. Bilateral pneumothorax occurred in three cases $(9 \%)$. One patient with previous PCP and pulmonary tuberculosis had haemopneumothorax, and one patient with $S$. aureus pneumonia and prior PCP suffered from pyopneumothorax.

Eight of the nine patients with current PCP $(89 \%)$ had diffuse bilateral and fairly symmetrical fine reticular heterogeneous opacities. Five of the 14 patients with previous PCP $(36 \%)$ and three of the nine with current PCP (33\%) had pneumatoceles throughout both lungs. Two of the 14 patients with previous PCP (14\%) and three of the nine with current PCP (33\%) had apical cysts detected on chest radiographs prior to PTX development. Two of the nine patients with current PCP (22\%) had subpleural cysts detected only by radiographs in the presence of PTX. Ten of the 13 patients with pulmonary tuberculosis $(77 \%)$ had coarse interstitial densities and five of the $13(38 \%)$ had hilar or mediastinal adenopathy. Bullae were observed in 10 of the 13 patients with pulmonary tuberculosis $(77 \%)$. Two of the 13 patients with tuberculosis $(15 \%)$ had a cavernous lesion of the right lower lobe. Three patients with prior PCP and active bacterial pneumonia had parenchymal infiltrates on chest radiographs. One patient with current PCP and Kaposi's pulmonary sarcoma had severe bilateral heterogeneous densities. CT scan, performed in 23 patients, documented the presence of cysts; in the upper lobes in 15 of the 23. Three patients $(9 \%)$ had normal chest radiographs prior to the development of PTX.

\section{Treatment and clinical outcome}

Five patients with asymptomatic small PTX were successfully treated with observation and oxygen. Thirty patients with moderate and large PTX were treated with thoracostomy drains, inserted with large bore $(36 \mathrm{~F})$ plastic intercostal chest tubes, connected either to an underwater seal or to suction. This therapeutic approach was successful in 20 out of 30 patients $(67 \%)$. Four of the 30 patients $(13 \%)$, in whom PTX failed to completely resolve with simple chest tube management, were treated with chemical pleurodesis (with bleomycin solution via the chest tube), and three of them (75\%) completely resolved. None of the patients needed surgical intervention. The average time required to achieve a successful response to any (including conservative) treatment was 11 days, ranging 3-18 days. Four patients developed a second episode of PTX, 5 months (as a median) after the first. Two of these patients suffered from bilateral and tension PTX.

The overall mortality was $23 \%$. In particular, seven patients died in the course of the first episode of PTX and two in a recurrence. Bilateral PTX was not significantly associated with hastened mortality, although the value was close to statistical significance $(p=0.06)$.

\section{Discussion}

The occurrence of PTX in HIV-infected patients was first reported in 1984 [1], and since then several other reports [2-5] have indicated that patients with AIDS are at increased risk of spontaneous PTX. Different causes have been implicated in the development of PTX in patients with AIDS, namely: $P$. carinii infection $[6,7,13-16]$; HIV itself, which can cause (probably through different mechanisms) a destructive action on the lung parenchyma, with alveolitis and "premature emphysema" [17]; and aerosolized pentamidine for PCP prophylaxis [8, 9]. It has been suggested that aerosolized pentamidine leaves the apical areas of the lung untreated, and, if PCP develops, there is a progressive apical disease with large subpleural cavities, which may rupture and cause PTX [8]. However, contrary to previous reports $[8,9]$, our data do not confirm the increased incidence of PTX in patients receiving prophylaxis with aerosolized pentamidine.

In the present study, the rate of PTX was 11.2 per 
1,000 hospital admissions for HIV infection. This rate greatly exceeds the overall incidence of spontaneous PTX in immunocompetent patients, which is estimated to be about 0.06 per 1,000 hospital admissions per year [4, 18]. In particular, the present study indicates that the episode prevalence of PTX in patients with PCP is 9.5\%, and $6.8 \%$ in those with pulmonary tuberculosis. The first percentage confirms the association, already reported [1, 4, 11], of spontaneous PTX with current or previous PCP in patients with AIDS. As a novel observation, the present study indicates an increased frequency of PTX in patients with AIDS and pulmonary tuberculosis. This result is further confirmed by the percentage of the population attributable risk, which indicates that more than $20 \%$ of PTX incidence in the AIDS population could be linked to the presence of pulmonary tuberculosis. Indeed, the association of pulmonary tuberculosis and PTX is not infrequently reported in immunocompetent hosts as a consequence of the rupture of subpleural blebs or a tuberculous cavity into the pleural space. However, these conditions do not seem to be frequent in HIVinfected patients, and they were responsible for development of PTX in only a few of the present patients. As a pathogenic mechanism, it is possible that $M$. tuberculosis, either directly or through the activation of pulmonary macrophages (possibly in association with HIV), may induce a chronic inflammatory reaction at the bronchiolar level, which causes obstruction, hyperinflation, progressive alveolar rupture and, finally, in some cases, PTX. Based on the results of our retrospective analysis, we can suggest that the increased rate of spontaneous PTX observed in HIV patients with pulmonary tuberculosis, independently from PCP or PCP prophylaxis, is the direct consequence of the growing number of HIV patients with tuberculosis observed worldwide. Prospective studies are, however, required to further confirm the association of pulmonary tuberculosis with PTX in patients with AIDS.

The statistically significant relationship of PTX and the presence of radiographic alterations of the lungs (cysts, pneumatoceles and bullae) in patients without PCP, could indicate that these lesions are important predictors of the risk of PTX. In fact, in association with the loss of interalveolar and anchoring septa in the lung periphery, the interstitial inflammatory and fibrotic reactions can cause cystic degenerative evolution, which predisposes the patients to formation of bullae or cavernous areas with subsequent spontaneous rupture and PTX.

Surprisingly, the mortality of the present patients with AIDS and PTX was lower than that indicated in previous reports [3, 19], and the presence of bilateral PTX was not associated with hastened mortality, although the value was close to statistical significance. Although it is difficult to explain these differences in mortality, it is possible to speculate that the variable distribution of opportunistic infections and neoplasms in the present study compared to previous reports [9, 19] could have influenced the mortality.

In conclusion, this $9 \mathrm{yr}$ retrospective survey confirms that pneumothorax is not infrequently observed in human immunodeficiency virus-infected patients, particularly in the setting of Pneumocystis carinii pneumonia, and it highlights a possible aetiological role of pulmonary tuberculosis in the development of pneumothorax. Due to the emerging importance of tuberculosis in human immunodeficiency virus-infected subjects, we recommend that pneumothorax should be considered in all patients with pulmonary tuberculosis who deteriorate acutely with worsening dyspnoea.

Acknowledgement: The authors are indebted to colleagues in the Department of Microbiology for their support.

\section{References}

1. Wollschager CM, Khan FA, Chitkara RK, Shivaram U. Pulmonary manifestations of the acquired immunodeficiency syndrome (AIDS). Chest 1984; 85: 197-202.

2. Byrnes TA, Breving JK, Yeoh CB. Pneumothorax in patients with acquired immunodeficiency syndrome. $J$ Thorac Cardiovasc Surg 1989; 98: 546-550.

3. Beers MF, Sohn M, Swartz M. Recurrent pneumothorax in AIDS patients with Pneumocystis pneumonia. Chest 1990; 98: 266-270.

4. Sepkowitz KA, Telzak EE, Gold JWM, et al. Pneumothorax in AIDS. Ann Intern Med 1991; 114: 455-459.

5. Coker RJ, Moss F, Peters B, et al. Pneumothorax in patients with AIDS. Respir Med 1993; 87: 43-47.

6. Feuerstein IM, Archer A, Pluda JM, et al. Thin-walled cavities, cysts, and pneumothorax in Pneumocystis carinii pneumonia: further observations with histopathologic correlation. Radiology 1990; 174: 697-702.

7. McClellan MD, Miller SB, Parsons PE, Cohn DL. Pneumothorax with Pneumocystis carinii pneumonia in AIDS. Chest 1991; 100: 1224-1228.

8. Renzi PM, Corbeil C, Chassè M, Braidy J, Matar N. Bilateral pneumothoraces hasten mortality in AIDS patients receiving secondary prophylaxis with aerosolized pentamidine. Chest 1992; 102: 491-496.

9. Newsome GS, Ward DJ, Pierce PF. Spontaneous pneumothorax in patients with acquired immunodeficiency syndrome treated with prophylactic aerosolized pentamidine. Arch Intern Med 1990; 150: 2167-2168.

10. Rossman MD, Mayock RL. Pulmonary tuberculosis. In: Schlossberg D, ed. Tuberculosis. New York, SpringerVerlag, 1994; pp. 95-106.

11. Nunn P, Mungai M, Nyawaya J, et al. The effect of human immunodeficiency virus type 1 on the infectiousness of tuberculosis. Tuber Lung Dis 1994; 75: 25-32.

12. Centers for Disease Control. 1993 revised classification system for HIV infection and expanded surveillance case definition for AIDS among adolescents and adults. MMWR Morb Mortal Wkly Rep 1992; 41 (RR17): 1-19.

13. Glatt AE, Chirgwin K. Pneumocystis carinii pneumonia in human immunodeficiency virus infected patients. Arch Intern Med 1990; 150: 271-279.

14. Goodman PC, Daley C, Mingei H. Spontaneous pneumothorax in AIDS patients with Pneumocystis carinii pneumonia. Am J Roentgenol 1986; 147: 29-31.

15. Finestone H, Goldfarb R, Ongseng F, Wasserman I, Garcia H. Scintigraphic pattern of pneumothorax complicating Pneumocystis carinii pneumonia in patients with AIDS. Clin Nucl Med 1990; 15: 566-568.

16. Metersky ML, Colt HG, Olson LK, Shanks TG. AIDSrelated spontaneous pneumothorax: risk factors and treatment. Chest 1995; 108: 946-951.

17. Kuhlman JE, Knowles MC, Fishman EK, Siegelman SS. Premature bullous pulmonary damage in AIDS: CT diagnosis. Radiology 1989; 173: 23-26.

18. Melton LJ, Hepper NC, Offord KP. Incidence of spontaneous pneumothorax in Olmsted County, Minessota, 1950 to 1974. Am Rev Respir Dis 1979; 120: 1379-1382.

19. Pinsk R, Rogers LF. Cystic parenchymal changes associated with spontaneous pneumothorax in an HIV-positive patients. Chest 1990; 97: 1471-1472. 